\title{
Expression of cyclooxygenase-2 in cervical cancer is associated with lymphovascular invasion
}

\author{
FRIEDERIKE HOELLEN ${ }^{1}$, ANNIKA WALDMANN ${ }^{2}$, CONSTANZE BANZ-JANSEN ${ }^{1}$, \\ ACHIM RODY ${ }^{1}$, MARIA HEIDE ${ }^{1}$, FRANK KÖSTER ${ }^{1}$, JULIKA RIBBAT-IDEL ${ }^{3}$, CHRISTOPH THORNS $^{3}$, \\ MAXIMILIAN GEBHARD ${ }^{4}$, MARTINA OBERLÄNDER ${ }^{5}$, JENS K. HABERMANN ${ }^{5}$ and MARC THILL ${ }^{6}$ \\ ${ }^{1}$ Department of Gynecology and Obstetrics, University of Lübeck and University Hospital Schleswig-Holstein, \\ Lübeck Campus, Lübeck D-23538; ${ }^{2}$ Institute for Social Medicine and Epidemiology, and ${ }^{3}$ Institute for Pathology, \\ University of Lübeck, Lübeck D-23538; ${ }^{4}$ Institute for Pathology, Hamburg D-22339; \\ ${ }^{5}$ Department of Surgery, Section for Translational Surgical Oncology and Biobanking, \\ University of Lübeck and University Hospital Schleswig-Holstein, Lübeck Campus, Lübeck D-23562; \\ ${ }^{6}$ Department of Gynecology and Obstetrics, Agaplesion Markus Hospital, Frankfurt D-60431, Germany
}

Received January 22, 2016; Accepted May 23, 2016

DOI: $10.3892 / \mathrm{ol} .2016 .4925$

\begin{abstract}
Cyclooxygenase-2 (COX-2) is associated with carcinogenesis and tumor progression. The current study analyzed the effect of COX-2 expression in patients with invasive squamous cervical cancer. Tissue samples from 123 cervical cancer patients were collected for a retrospective analysis using immunohistochemistry (IHC) with an antibody against COX-2. The clinical and survival data of the patients were analyzed. Positive staining for COX-2 (defined as an immunoreactivity score of $\geq 4$ ) was detected in 28 patients $(23 \%)$, with significantly higher percentages of staining in tumor cells compared with peritumoral stroma cells $(\mathrm{P}<0.001)$. COX-2 expression was significantly associated with lymphovascular space invasion (LVSI; $\mathrm{P}=0.017$ ). The association of COX-2 expression with LVSI suggests a possible effect of COX-2 on tumor progression in cervical cancer. Further studies including larger patient collectives are required in order to perform analyses of clinical subgroups and patient survival.
\end{abstract}

\section{Introduction}

Prostaglandins are active lipid compounds that are synthesized in mammals from free arachidonic acid by two isoenzymes, cyclooxygenase (COX)-1 and -2. As a result of

Correspondence to: Dr Friederike Hoellen, Department of Gynaecology and Obstetrics, University of Lübeck and University Hospital Schleswig-Holstein, Lübeck Campus, 160 Ratzeburger Allee, Lübeck D-23538, Germany

E-mail: friederike.hoellen@uksh.de

Key words: cervical cancer, cyclooxygenase-2, lymphangiosis, lymphovascular space invasion their structural heterogeneity, COX-1 and -2 have diverse biological functions. Prostaglandins, in addition to their inflammatory activity, are involved in carcinogenesis and tumor growth (1). In a variety of tumor entities, inflammation and its underlying molecular processes have been thoroughly investigated as a critical component of tumor progression. Increasing evidence indicates that the tumor microenvironment, which is characterized by inflammatory cells, participates in the neoplastic process; it fosters proliferation, survival and migration, conducted by receptors for invasion, migration and metastasis (2). COX-2, a key enzyme related to prostaglandin metabolism, is crucial in the neoplastic process and serves as a prognostic factor for malignancy (3). Furthermore, COX-2 has been shown to be associated with carcinogenesis, particularly with neoangiogenesis and tumor progression (4-9). Increased COX-2 expression has been demonstrated in epithelial cancer cells, including colon, gastric, esophageal, lung, hepatic, pancreatic, prostate, ovarian, breast and cervical cancers (4-14). In cervical cancer, human papillomavirus (HPV) is the major risk factor for carcinogenesis. Thus, the concept of elevated COX-2, in a milieu of viral inflammation, as a trigger for carcinogenesis in cervical cancer is evident. Activation of the COX-2/prostaglandin E2 pathway by viral oncogenic proteins (HPV subtype 16, oncoproteins E5, E6 and E7) is associated with an increase in COX-2 expression (15).

COX-2 expression was previously demonstrated to be increased in preinvasive lesions, and early and advanced cervical cancer, and its upregulation is particularly present in patients with metastases and poor prognosis. According to clinical data, certain studies have reported that high levels of COX-2 expression are associated with a higher incidence of parametrial invasion and lymph node metastases in cervical cancer (15). Furthermore, epidemiological data suggest that COX-2 inhibitors may have an effect on carcinogenesis (16).

In the present retrospective study, the clinical data, tumor characteristics and survival data of cervical cancer patients 
were evaluated and analyzed with respect to the presence and strength of COX-2-expression.

\section{Patients and methods}

Patients. Between 2003 and 2011, cervical cancer tumor sections were collected from 123 patients who underwent radical hysterectomy and at least one lymph node sampling for invasive squamous cervical cancer at the University Medical Center Schleswig-Holstein, Campus Lübeck (Lübeck, Germany). Patients with adenocarcinoma and patients who underwent neoadjuvant radiation or neoadjuvant chemotherapy were excluded from the analysis.

For all 123 patients, the hospital records were retrospectively reviewed, and data regarding patient characteristics, pathological diagnosis and adjuvant therapy were extracted, in addition to the date of the final follow-up examination or date of mortality. Tumor-Node-Metastasis (TNM) and Internation Federation of Gynecology and Obstetrics (FIGO) stages and lymphangiosis carcinomatosa [lymphovascular space invasion (LVSI)] status were derived from the original histopathological reports. In 7 patients of FIGO stages III and IV who were included, up-staging occurred following surgery or emergency simple hysterectomy, performed for heavy bleeding, with lymph node sampling.

All patients provided written informed consent in order to allow for retrospective data analysis on the basis of an anonymized dataset. The study protocol was approved by the Regional Ethics Committee.

Tissue analysis. Resected cervical cancer tissue, representing tumor and corresponding peritumoral stromal tissue were implemented into tissue microarrays (TMAs) as described $(17,18)$. Briefly, small biopsies were retrieved from selected regions of paraffinized donor tissue blocks using hollow stainless-steel needles. Cores were inserted into a standard-sized recipient array block. The TMAs were constructed using a semi-automated arrayer (TMArrayer; Pathology Devices, Inc., Westminster, MD, USA). Four replicate cores were taken per sample. In order to include all samples, a total of four TMA blocks was constructed.

Multiple 4- $\mu \mathrm{m}$ sections were cut with a Micron microtome as described (18) and stained by specific antibodies for immunohistochemical (IHC) analysis. A monoclonal mouse anti-human COX-2 antibody (clone CX-294; \#M3617; Dako, Hamburg, Germany) was used at a dilution of 1:100 on TMAs, according to the manufacturer's recommendations. Liver tissue and peritumoral cervical stromal tissue served as controls. Representative and viable tissue sections were reviewed. The IHC staining of the samples was evaluated by a gynecological histopathologist using the immunoreactivity score (IRS) according to Remmele and Stegner (19); this evaluation system includes staining intensity and quantitative count of positive cells. When assessing a given core, the observer was blinded to the scores of other variables and to outcome. Samples were interpreted as COX-2-positive if the IRS was $\geq 4$.

Statistical analysis. The statistical analysis was conducted using SPSS software (version 22; IBM SPSS, Armonk, NY, USA). Quantitative data are described as the means with

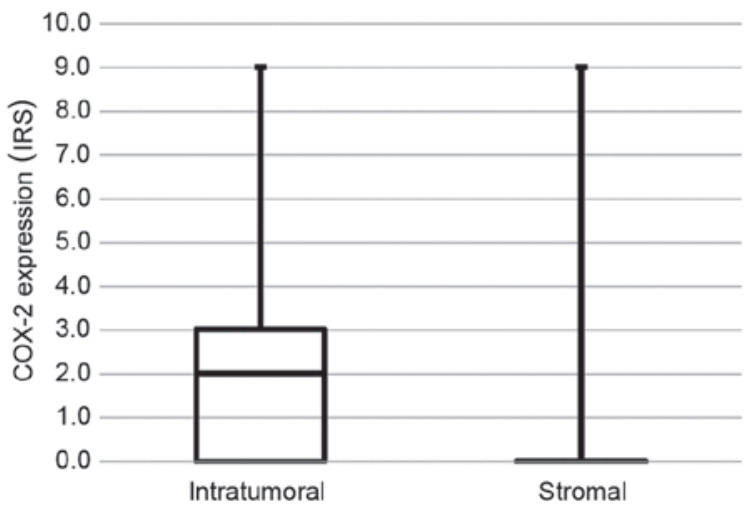

Figure 1. COX-2 expression in tumor tissue and peritumoral stroma $(\mathrm{P}<0.001)$. COX-2, cyclooxygenase-2; IRS, immunoreactivity score.

standard deviations (SD). In the case of age at diagnosis, age ranges are also reported. Qualitative data are described with absolute and relative frequencies. Student's $t$-test was used to determine differences regarding the age of patients between groups with and without COX-2 expression. The $\chi^{2}$ and Fisher's exact tests were used to examine differences regarding molecular markers and clinicopathological characteristics between patient groups with and without COX-2 expression. Logistic regression was conducted to establish the main factors responsible for lymph node involvement with COX-2 expression and lymphangiosis as predictors. The odds ratios (OR) and 95\% confidence intervals (95\% CI) ascertained by the logistic regression are reported.

Differences in survival between cervical cancer patients with and without COX-2 expression were tested with the log-rank test and Kaplan-Meier survival curves were plotted. A Cox proportional hazards model was computed to reveal significant determinants of mortality following cervical cancer. The significance threshold used for survival analyses was $\mathrm{P}<0.05$.

\section{Results}

Patient characteristics. An overview of patient characteristics is given in Table I. The majority of patients (94\%) were FIGO stage I and II, according to the inclusion criteria (radical hysterectomy). Nearly $95 \%$ of the patients had T stage 1 or 2 tumors, and were free of distant metastases. In accordance with the high proportion of G3 tumors, $~ 50 \%$ of all patients received adjuvant chemotherapy and $61 \%$ received adjuvant radiotherapy. The expression of COX-2 ranged from IRS 0 to 9 in tumor samples from patients with cervical cancer, with a median IRS of 2 (Fig. 1). In the peritumoral stroma, the expression of COX-2 was nearly undetectable $(\mathrm{P}<0.001)$.

COX-2 and clinicopathological parameters. Using a cut-off score of IRS $\geq 4$ to determine positive expression of COX-2, $23 \%$ of the cervical cancer patients $(n=28)$ were classified as COX-2-positive and $77 \%(\mathrm{n}=95)$ were negative (Fig. 2). In a bivariate analysis, patients with and without COX-2 expression did not differ regarding tumor and clinicopathological characteristics, adjuvant therapy or smoking status. 
Table I. Description of 123 patients with cervical cancer with respect to COX-2 expression.

\begin{tabular}{|c|c|c|c|}
\hline Variable & Overall & COX-2-negative & COX-2-positive \\
\hline Total patients, n (\%) & 123 & 95 (77.2) & $28(22.8)$ \\
\hline \multicolumn{4}{|l|}{ Age (years) at surgery } \\
\hline Mean \pm SD & $52.4 \pm 12.8$ & $51.5 \pm 12.4$ & $55.5 \pm 13.9$ \\
\hline Range & $25-79$ & $25-75$ & $34-79$ \\
\hline \multicolumn{4}{|l|}{ T stage, $\mathrm{n}\left(\%^{\mathrm{a}}\right)$} \\
\hline урТ0 & $2(1.6)$ & $2(2.1)$ & $0(0.0)$ \\
\hline $\mathrm{T} 1$ & $77(63.1)$ & $60(63.8)$ & $17(60.7)$ \\
\hline $\mathrm{T} 2$ & $38(31.1)$ & $29(30.9)$ & $9(32.1)$ \\
\hline $\mathrm{T} 3$ & $3(2.5)$ & $2(2.1)$ & $1(3.6)$ \\
\hline $\mathrm{T} 4$ & $2(1.6)$ & $1(1.1)$ & $1(3.6)$ \\
\hline Tx (unknown) & 1 & 1 & 0 \\
\hline \multicolumn{4}{|l|}{$\mathrm{N}$ stage, $\mathrm{n}\left(\%^{\mathrm{a}}\right)$} \\
\hline N0 & $93(75.6)$ & 75 (78.9) & $18(64.3)$ \\
\hline N1 & $30(24.4)$ & $20(21.1)$ & $10(35.7)$ \\
\hline \multicolumn{4}{|l|}{ M stage, n $\left(\%^{\mathrm{a}}\right)$} \\
\hline M0 & 118 (95.9) & $92(96.8)$ & $26(92.9)$ \\
\hline M1 & $5(4.1)$ & $3(3.2)$ & $2(7.1)$ \\
\hline \multicolumn{4}{|l|}{ Hemangiosis, $\mathrm{n}\left(\%^{\mathrm{a}}\right)$} \\
\hline Negative & $54(96.4)$ & $38(95.0)$ & $16(100.0)$ \\
\hline Positive & $2(3.6)$ & $2(5.0)$ & $0(0.0)$ \\
\hline Unknown & 67 & 55 & 12 \\
\hline \multicolumn{4}{|l|}{ Lymphangiosis, $\mathrm{n}\left(\%^{\mathrm{a}}\right)$} \\
\hline Negative & $47(68.1)$ & $39(78.0)$ & $8(42.1)$ \\
\hline Positive & $22(31.9)$ & $11(22.2)$ & $11(57.9)$ \\
\hline Unknown & 54 & 45 & 9 \\
\hline \multicolumn{4}{|l|}{ Grade, $\mathrm{n}\left(\%^{\mathrm{a}}\right)$} \\
\hline G1 & $2(1.7)$ & $2(2.1)$ & $0(0.0)$ \\
\hline $\mathrm{G} 2$ & $56(46.7)$ & $43(45,7)$ & $13(50.0)$ \\
\hline G3 & $62(51.7)$ & $49(52,1)$ & $13(50.0)$ \\
\hline Gx (unknown) & 3 & & 2 \\
\hline \multicolumn{4}{|l|}{ FIGO stage, n $\left(\%^{\mathrm{a}}\right)$} \\
\hline $\mathrm{I}$ & $77(62.6)$ & $60(63.2)$ & $17(60.7)$ \\
\hline II & $39(31.7)$ & $30(31.6)$ & $9(32.1)$ \\
\hline III & $5(4.1)$ & $4(4.2)$ & $1(3.6)$ \\
\hline IV & $2(1.6)$ & $1(1.1)$ & $1(3.6)$ \\
\hline \multicolumn{4}{|c|}{ History of smoking, $\mathrm{n}\left(\%^{\mathrm{a}}\right)$} \\
\hline Yes & $51(41.5)$ & $41(43.2)$ & $10(35.7)$ \\
\hline No & $72(58.5)$ & $54(56.8)$ & $18(64.3)$ \\
\hline \multicolumn{4}{|c|}{ Chemotherapy administered, $\mathrm{n}\left(\%^{\mathrm{a}}\right)$} \\
\hline Yes & $59(49.2)$ & $43(46.2)$ & $16(59.3)$ \\
\hline No & $61(50.8)$ & $50(53,8)$ & $11(40,7)$ \\
\hline Unknown & 3 & 1 & 2 \\
\hline \multicolumn{4}{|c|}{ Radiotherapy administered, $\mathrm{n}\left(\%^{\mathrm{a}}\right)$} \\
\hline Yes & $75(61.0)$ & $58(61.1)$ & $17(60.7)$ \\
\hline No & $48(39.0)$ & $37(38.9)$ & $11(39.3)$ \\
\hline
\end{tabular}

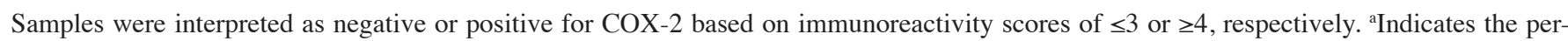
centage of patients with known status for each variable, with respect to COX-2 expression group. COX-2, cyclooxygenase-2; SD, standard deviation; T, tumor; N, node; M, metastasis; FIGO, International Federation of Gynecology and Obstetrics; yp 10, in histology no more tumor cells detectable after neoadjuvant chemotherapy. 


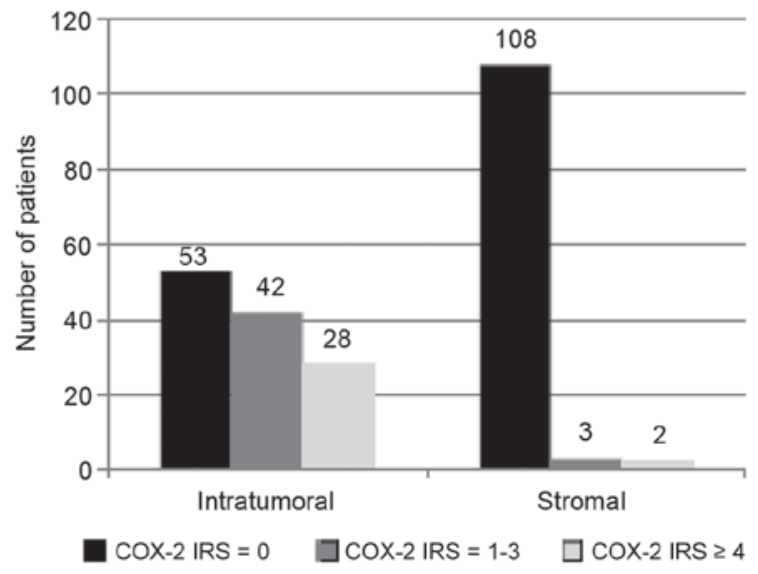

Figure 2. Levels of COX-2 expression in tumor tissue and peritumoral stroma. COX-2, cyclooxygenase-2; IRS, immunoreactivity score.

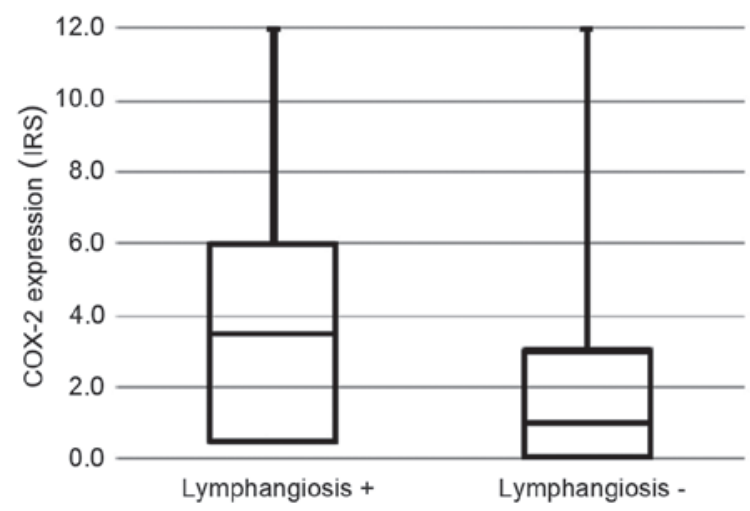

Figure 3. COX-2 expression in patients with and without lymphangiosis $(\mathrm{P}=0.017)$. COX-2, cyclooxygenase-2; IRS, immunoreactivity score.

COX-2 and lymphovascular invasion.COX-2-positive patients were significantly more likely to experience lymphangiosis (Table I). Fig. 3 shows the distribution of COX-2 expression in all patients with and without lymphangiosis. Furthermore, patients with lymphangiosis and COX-2 expression more often developed lymph node metastases $(6 / 11,55 \%)$ compared with patients without lymphangiosis and without COX-expression $(5 / 39,13 \%, \mathrm{P}=0.003)$. The combined data for lymphangiosis, COX-2 expression and lymph node involvement was available for 50 patients. In the logistic regression analysis, only lymphangiosis was found to increase the risk of lymph node involvement significantly (OR, 8.23; 95\% CI, 2.29-29.60), while COX-2 expression showed no significant effect (OR, $0.99 ; 95 \%$ CI, 0.26-3.83).

Follow-up. Data were available for 115 out of 123 patients. During a mean follow-up period of 5 years (SD, 3.2 years), 21 mortalities (18.3\%) occurred among the cervical cancer patients. During the first and the second years subsequent to surgery, 3 patients died each year, and another 6 patients died during the third year of follow-up. Among the 26 patients with COX-2 expression, 6 (23.1\%) mortalities occurred, while 15 (17.0\%) occurred and among the patients without COX-2 expression $(\mathrm{P}=0.486)$. Fig. 4 shows survival curves for patients with and without COX-2 expression (log-rank test, $\mathrm{P}=0.717$ ).

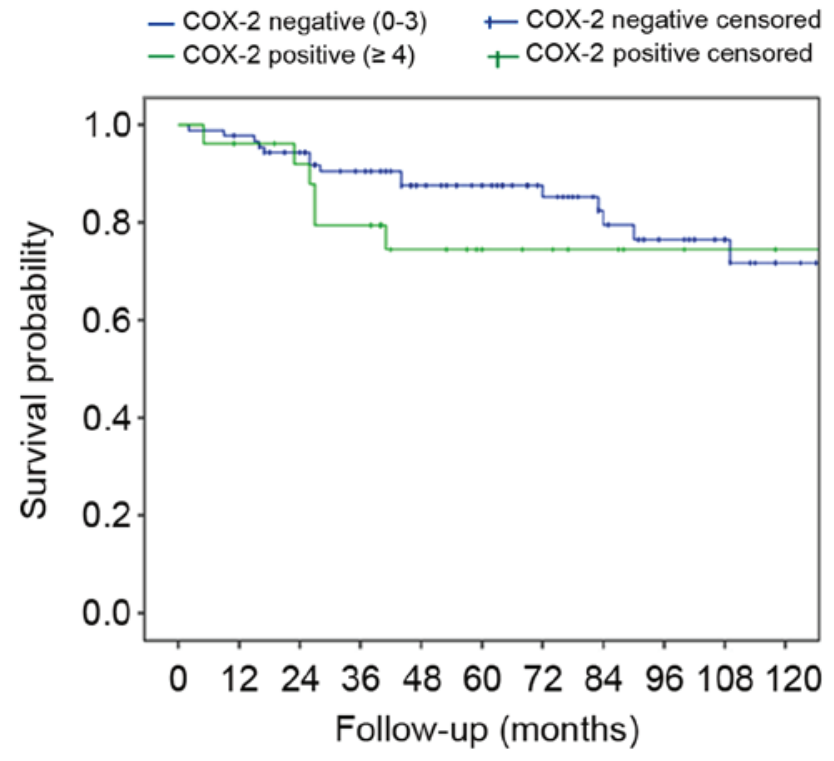

Figure 4. Survival curves of patients with and without COX-2 expression (log-rank test, $\mathrm{P}=0.717$ ). COX-2, cyclooxygenase-2.

\section{Discussion}

Promising results regarding COX-2 as a trigger of carcinogenesis in preinvasive cancerous lesions in various tumor entities suggest the potential of COX-2 for targeted therapy in cervical cancer. In a recent publication, the authors reported a potential role for COX-2 inhibitors in drug repositioning in cervical cancer (16). Apart from a direct anticancer effect, COX-2 inhibitors increase the sensitivity of cancer cells to drugs and radiotherapy. In patients with advanced recurrent cervical cancer, a current clinical trial of tegafur-uracil (UFT) in combination with cyclophosphamide and the COX-2 inhibitor celecoxib is performed. In vitro studies showed that COX-2 might play a role in acquisition of resistance to taxanes and COX-2 inhibitors enhance the anticancer effects of cisplatin and paclitaxel. Furthermore, COX-2 inhibitors may increase radiosensitivity.

In the present retrospective study of 123 patients, a significant association was identified between the expression of COX-2 and lymphangiosis (LVSI). No associated was detected between the expression of COX-2 and tumor stage or grade. To date, COX-2 analysis scoring systems have been extremely heterogeneous. In the majority of cases, quantitative estimation was conducted. In order to establish a reliable semi-quantitative scoring system, IRS according to Remmele and Stegner (19) was used in the present study; this system takes into account the percentage of stained cells as well as the intensity of staining.

In the current patient collective, $43 \%$ of tumor tissues were COX-2-negative (IRS 0), and 57\% showed at least a weak expression (IRS 1-12). Using a cut-off of IRS $\geq 4$ according to the IRS scoring system, $23 \%$ of samples were determined to be COX-2-positive. Dursun et al (20) reported a percentage of 55.2\% COX-2-positive squamous cervical cancers using a scoring system based on the percentage of positive cells, with a cut-off at $50 \%$. Manchana et al (21) described a patient cohort with 40.6\% COX-2-positive, FIGO stage IB, squamous 
cervical cancers. In that analysis, COX-2 immunoreactivity was estimated positive if $>50 \%$ of the cells were stained. In a bivariate analysis, the present study identified no association of COX-2 expression with tumor grade.

Previously reported data suggest an association between the expression of COX-2 and lymph node metastases (22-25), FIGO stage (26), tumor size (26), and parametrial infiltration $(21,27,28)$; however the data are inconsistent. Tumor grade has not yet been shown to be correlated with the expression of $\mathrm{COX}-2$, based on the current literature. Certain of these studies included adenocarcinoma or tissues that had been subjected to neoadjuvant treatment. Therefore, the data may be not fully reliable with regard to cervical cancers.

In our bivariate analysis, no association could be identified between COX-2 expression and tumor grade. To the best of our knowledge, this is in line with the current literature. For example, Chen et al (29) reported increased expression of COX-2 in well-differentiated cervical carcinoma. However, the authors investigated adenocarcinoma of the uterine cervix, which must be clearly separated from squamous carcinoma.

None of tumor size, FIGO stage, distant metastases or parametrial infiltration were associated with COX-2 expression in the current patient collective. Some studies found a correlation of COX-2 with tumor size or tumor/stroma relation and FIGO stage $(20,26)$, others did not $(21,30,31)$. Three studies reporting an association of COX-2 with parametrial infiltration were identified $(21,22,27)$, however various studies did not support a correlation of COX-2 overexpression and parametrial infiltration $(21,20,29)$. Furthermore, when patient characteristics were analyzed, age was not associated with COX-2 expression. With the exception of one study (30), this observation is in line with those reported in the relevant literature $(21,26)$.

Our finding of a significant association of the expression of COX-2 with lymphangiosis (LVSI) was also reported by previous studies from other groups. LVSI has been identified as an independent predictor of lymph node metastases in cervical cancer in a multivariate analysis (32), and the association of COX-2 expression and LVSI has been demonstrated in various studies $(20,31,33,34)$. Although Manchana et al (21) did not find elevated COX-2 expression in LVSI, COX-2 was $100 \%$ positive in patients with lymph node metastases in this study. Analyses of the association between lymph node metastases and COX-2 overexpression have yielded varying results. Notably, in two studies, COX-2 expression was only associated with lymph node metastases when LVSI occurred simultaneously $(31,31)$. In one of those studies, COX-2 expression was associated with lymph node metastasis in cases with LVSI on multivariate analysis (not significant, $\mathrm{P}=0.068$ ) (31). In the present patient collective, COX-2 expression did not correlate significantly with lymph node metastasis $(\mathrm{P}=0.112)$. While certain currently available data suggest an association between COX-2 expression and lymph node metastases (22-25), others do not $(20,26,35,36)$. The present study also investigated the expression of COX-2 according to lymph node metastasis in patients with LVSI. Patients with lymphangiosis and positive reactivity for COX-2 more often developed lymph node metastases $(55 \%)$ than patients without lymphangiosis and without COX-2 expression (13\%; $\mathrm{P}=0.003)$. However, if the association is further explored by means of logistic regression analysis, only lymphangiosis increases the risk of lymph node involvement significantly, while COX-2 expression alone has no effect on lymph node metastases.

According to the present findings and data from the literature, COX-2 expression may be crucial in cervical cancer tumor progression in patients with LVSI. To the best of our knowledge, this association has not been identified in other tumor entities so far.

Survival did not differ significantly between patients with COX-2 expression (23.1\% mortality rate) and patients without COX-2 expression (17.0\% mortality rate; $\mathrm{P}=0.486)$. Survival curves for patients with and without COX-2 expression (Fig. 4) initially revealed a tendency towards better survival in COX-2-negative patients; however, this observation was not significant (log-rank, $\mathrm{P}=0.717$ ). The data of the present patient collective must be interpreted with caution due to the small collective and low mortality rate. Multivariate regression analysis (Cox proportional hazards model) did not reveal any significant factors for survival (lymphangiosis, $\mathrm{P}=0.74$; $\mathrm{COX}-2$, $\mathrm{P}=0.357$; FIGO, $\mathrm{P}=0.104$; grade, $\mathrm{P}=0.364$; age, $\mathrm{P}=0.057$ ). These findings are corroborated by certain authors, who reported a decline in overall survival (OS) in COX-2-positive cervical cancer $(26,27,37)$ and poor response to radiotherapy and chemotherapy $(27,37,38)$.

The absolute 5-year OS rate for cervical cancer in Germany for all stages is $\sim 65 \%$. In the present patient collective, the 5 -year OS rate was $81.7 \%$. In comparison to the tumor stage incidence of cervical cancer in Germany at initial diagnosis (T1, 61\%; T2, 25\%; T3, 8\%; T4, 6\%) (39), the present patient collective showed a different distribution, with $94.2 \%$ of patients in $\mathrm{T} 1$ and $\mathrm{T} 2$. This difference in stages at first diagnosis is due to the inclusion criteria employed in the present study: The majority of T3 and T4 tumors were not included as those patients received primary radiochemotherapy according to advanced cancer stage, and not hysterectomy and lymphadenectomy, which would be in line with our inclusion criteria. Consequently, this collective had a better 5-year OS rate in comparison to general German cervical cancer patients (Cancer in Germany 2015. Available at: http://www. rki.de/DE/Content//Pressemitteilungen/Presse/2015/11_2015). The mean age of our patient collective was 52.4 years (Table I), congruent with German epidemiological data of cervical cancer (53 years) (39).

In summary, our results support the hypothesis of COX-2 involvement in LVSI and consequently in lymph node metastases. In view of the small patient collective and the limited evidence being available, further studies with larger patient collectives, adequate statistical power for subgroup analyses and long-term follow-up and survival data are required. The potential of COX-2 inhibitors for inhibition of tumor progression in early-stage cervical cancer may be a possible target for future targeted therapies. In terms of surgical therapies, COX-2 expression could influence the indication for the extent of lymph node dissection. Furthermore, COX-2 as a prognostic marker could be part of decision-making for adjuvant therapies.

\section{References}

1. Murakami M and Kudo I: Recent advances in molecular biology and physiology of the prostaglandin E2-biosynthetic pathway. Prog Lipid Res 43: 3-35, 2004. 
2. Coussens LM and Werb Z: Inflammation and cancer. Nature 420 $860-867,2002$

3. Herschman HR: Prostaglandin synthase 2. Biochim Biophys Acta 1299: 125-140, 1996.

4. Ristimäki A, Sivula A, Lundin J, Lundin M, Salminen T, Haglund C, Joensuu $\mathrm{H}$ and Isola J: Prognostic significance of elevated cyclooxygenase-2 expression in breast cancer. Cancer Res 62: 632-635, 2002

5. Rozic JG, Chakraborty C and Lala PK: Cyclooxygenase inhibitors retard murine mammary tumor progression by reducing tumor cell migration, invasiveness and angiogenesis. Int $\mathrm{J}$ Cancer 93: 497-506, 2001.

6. Swami S, Krishnan AV, Moreno J, Bhattacharyya RB, Peehl DM and Feldman D: Calcitriol and genistein actions to inhibit the prostaglandin pathway: Potential combination therapy to treat prostate cancer. J Nutr 137 (Suppl 1): S205-S210, 2007.

7. Subbaramaiah K and Dannenberg AJ: Cyclooxygenase 2: A molecular target for cancer prevention and treatment. Trends Pharmacol Sci 24: 96-102, 2003.

8. Ali-Fehmi R, Morris RT, Bandyopadhyay S, Che M, Schimp V, Malone JM Jr and Munkarah AR: Expression of cyclooxygenase-2 in advanced stage ovarian serous carcinoma: Correlation with tumor cell proliferation, apoptosis, angiogenesis and survival. Am J Obstet Gynecol 192: 819-825, 2005.

9. Ranger GS, Thomas V, Jewell A and Mokbel K: Elevated cyclooxygenase- 2 expression correlates with distant metastases in breast cancer. Anticancer Res 24: 2349-2351, 2004.

10. Eberhart CE, Coffey RJ, Radhika A, Giardiello FM, Ferrenbach S and DuBois RN: Up-regulation of cyclooxygenase 2 gene expression in human colorectal adenomas and adenocarcinomas. Gastroenterology 107: 1183-1188, 1994.

11. Sinicrope FA, Lemoine M, Xi L, Lynch PM, Cleary KR, Shen Y and Frazier ML: Reduced expression of cyclooxygenase 2 proteins in hereditary nonpolyposis colorectal cancers relative to sporadic cancers. Gastroenterology 117: 350-358, 1999.

12. Ristimäki A, Jaatinen R and Ritvos O: Regulation of prostaglandin F2 alpha receptor expression in cultured human granulosa-luteal cells. Endocrinology 138: 191-195, 1997.

13. Zimmermann KC, Sarbia M, Weber AA, Borchard F, Gabbert HE and Schrör K: Cyclooxygenase-2 expression in human esophageal carcinoma. Cancer Res 59: 198-204, 1999.

14. Heinonen PK and Metsä-Ketelä T: Prostaglandin and thromboxane production in ovarian cancer tissue. Gynecol Obstet Invest 18: 225-229, 1984

15. Parida $\mathrm{S}$ and Mandal M: Inflammation induced by human papillomavirus in cervical cancer and its implication in prevention. Eur J Cancer Prev 23: 432-448, 2014

16. Banno K, Iida M, Yanokura M, Irie H, Masuda K, Kobayashi Y, Tominaga E and Aoki D: Drug repositioning for gynecologic tumors: A new therapeutic strategy for cancer. Scientific World Journal 2015: 341362, 2015.

17. Braunschweig T, Chung JY and Hewitt SM: Tissue microarrays: Bridging the gap between research and the clinic. Expert Rev Proteomics 2: 325-336, 2005.

18. Oberländer M, Alkemade H, Bünger S, Ernst F, Thorns C, et al: A 'waterfall' transfer-based workflow for improved quality of tissue microarray construction and processing in breast cancer research. Pathol Oncol Res 20: 719-726, 2014.

19. Remmele W and Stegner HE: Recommendation for uniform definition of an immunoreactive score (IRS) for immunohistochemical estrogen receptor detection (ER-ICA) in breast cancer tissue. Pathologe 8: 138-140, 1987 (In German).

20. Dursun P, Yuce K, Usubutun A and Ayhan A: Cyclooxygenase-2 expression in cervical intraepithelial neoplasia III and squamous cell cervical carcinoma, and its correlation with clinicopathologic variables. Int J Gynecol Cancer 17: 164-173, 2007.

21. Manchana T, Triratanachat S, Sirisabya N, Vasuratna A, Termrungruanglert $\mathrm{W}$ and Tresukosol D: Prevalence and prognostic significance of COX-2 expression in stage IB cervical cancer. Gynecol Oncol 100: 556-560, 2006.

22. Kim HJ, Wu HG, Park IA and Ha SW: High cyclooxygenase-2 expression is related with distant metastasis in cervical cancer treated with radiotherapy. Int J Radiat Oncol Biol Phys 55: $16-20,2003$.

23. Kim JJ, Wang J, Bambra C, Das SK, Dey SK and Fazleabas AT: Expression of cyclooxygenase-1 and -2 in the baboon endometrium during the menstrual cycle and pregnancy. Endocrinology 140: 2672-2678, 1999.
24. Ryu HS, Chang KH, Yang HW, Kim MS, Kwon HC and Oh KS: High cyclooxygenase-2 expression in stage IB cervical cancer with lymph node metastasis or parametrial invasion. Gynecol Oncol 76: 320-325, 2000

25. Pyo H, Kim YB, Cho NH, Suh CO, Park TK, Yun YS and Kim GE: Coexpression of cyclooxygenase-2 and thymidine phosphorylase as a prognostic indicator in patients with FIGO stage IIB squamous cell carcinoma of uterine cervix treated with radiotherapy and concurrent chemotherapy. Int J Radiat Oncol Biol Phys 62: 725-732, 2005.

26. Ferrandina G, Lauriola L, Distefano MG, Zannoni GF, Gessi M, Legge F, Maggiano N, Mancuso S, Capelli A, Scambia G and Ranelletti FO: Increased cyclooxygenase-2 expression is associated with chemotherapy resistance and poor survival in cervical cancer patients. J Clin Oncol 20: 973-981, 2002.

27. Ferrandina G, Lauriola L, Zannoni GF, Distefano MG, Legge F, Salutari V, Gessi M, Maggiano N, Scambia G and Ranelletti FO: Expression of cyclooxygenase-2 (COX-2) in tumour and stroma compartments in cervical cancer: Clinical implications. Br J Cancer 87: 1145-1152, 2002.

28. Kim HJ, Sohn TS, Lee KT, Lee JK, Paik SW and Rhee JC: Expression of cyclooxygenase-2 and its correlation with clinicopathologic factors of ampulla of vater cancer. J Korean Med Sci 18: 218-224, 2003

29. Chen YJ, Wang LS, Wang PH, Lai CR, Yen MS, Ng HT and Yuan CC: High cyclooxygenase-2 expression in cervical adenocarcinomas. Gynecol Oncol 88: 379-385, 2003.

30. Giordano G, D'Adda T, Dal Bello B, Brigati F, Bersiga A, Campanini N, Berretta R, Rocco A and Merisio C: Clinicopathologic implications of the epidermal growth factor receptor cyclooxygenase 2 expression and human papillomavirus status in squamous cell carcinoma of the uterine cervix in the elderly. Int J Gynecol Cancer 21: 337-348, 2011

31. Khunamornpong S, Settakorn J, Sukpan K, Srisomboon J, Ruangvejvorachai P, Thorner PS and Siriaunkgul S: Cyclooxygenase-2 expression in squamous cell carcinoma of the uterine cervix is associated with lymph node metastasis. Gynecol Oncol 112: 241-247, 2009

32. Wang W, Jia HL, Huang JM, Liang YC, Tan H, Geng HZ, Guo LY and Yao SZ: Identification of biomarkers for lymph node metastasis in early-stage cervical cancer by tissue-based proteomics. Br J Cancer 110: 1748-1758, 2014.

33. Settakorn J, Khunamornpong S, Sukpan K, Srisomboon J and Siriaungkul S: Cyclooxygenase-2 expression in cervical squamous cell carcinoma: The significance of expression in neoplastic cells within the lymphovascular space. Asian Pac J Cancer Prev 10: 1011-1014, 2009.

34. Bandyopadhyay R, Chatterjee U, Mondal SK, Nag D and Sinha SK: A study on expression pattern of cyclooxygenase-2 in carcinoma of cervix. Indian J Pathol Microbiol 54: 695-699, 2011.

35. Kim JH and Kim SJ: Overexpression of microRNA-25 by withaferin A induces cyclooxygenase-2 expression in rabbit articular chondrocytes. J Pharmacol Sci 125: 83-90, 2014.

36. Ferrandina G, Ranelletti FO, Legge F, Lauriola L, Poerio A, Zannoni GF, Smaniotto D, Margariti PA, Macchia G and Scambia G: Cyclooxygenase-2 (COX-2) expression in locally advanced cervical cancer patients undergoing chemoradiation plus surgery. Int J Radiat Oncol Biol Phys 55: 21-27, 2003.

37. Lindström AK, Tot T, Stendahl U, Syrjänen S, Syrjänen K and Hellberg D: Discrepancies in expression and prognostic value of tumor markers in adenocarcinoma and squamous cell carcinoma in cervical cancer. Anticancer Res 29: 2577-2578, 2009.

38. Gaffney DK, Holden J, Davis M, Zempolich K, Murphy KJ and Dodson M: Elevated cyclooxygenase-2 expression correlates with diminished survival in carcinoma of the cervix treated with radiotherapy. Int J Radiat Oncol Biol Phys 49: 1213-1217, 2001

39. Robert Koch Institute. Neue Daten zu Krebs in Deutschland. http://www.rki.de/DE/Content/Service/Presse/Pressemitteilungen/2015/11_2015.html Accessed July 1, 2016. (In German). 